\title{
Managed Care Pharmacy Practice at the Texas Tech University Health Sciences Center School of Pharmacy
}

A cademic Health Sciences Centers (AHSCs) are not immune to the cost-containment pressures of a changing health care financing system. For state-funded health professions programs, the expanding gap between state funding and the cost of education, and the conversion of traditional AHSC patient care markets to competitive-bid managed care, are cause for serious concern about the future viability of health professions education.

A solution advocated by some AHSCs is the creation of business practice units to form practice partnerships with public and private business entities. The business relationship from the perspective of the AHSC is to provide the patient care expertise of its various educational programs in return for access to patients. The business partners receive a highly integrated educational and patient care delivery system for a competitive cost. These arrangements are "win-win" for all parties.

In 1995, the School of Pharmacy at Texas Tech University formed a managed care business unit primarily to support a comparable organization at the AHSC called Managed Health Care (MHC). The new pharmacy business unit called Managed Health Care Pharmacy Services (MHCPS) was organized to provide clinical services, disease management programs, pharmacy benefits management, and administrative oversight for contract drug distribution services, depending on the needs of the business partner. The School of Pharmacy carefully designed the mission and operational plan of the MHCPS to ensure that the educational and service needs of the faculty and students were a prominent component of any business relationship.

The first managed care contracts for pharmacy services were signed with the Texas Department of Criminal Justice (TDCJ) and the Texas Youth Commis- sion (TYC) correctional and youth offender facilities in West Texas. The TDCJ and TYC have similar arrangements with the University of Texas Medical Branch for medical/psychiatric care and the University of Houston for pharmaceutical care for the eastern part of the state.

Several articles have been written that describe the relationships for this staff-model HMO arrangement. ${ }^{1-3}$ This article focuses on some of the unique aspects of a pharmacy school-based managed care organization and its role within a statewide, integrated, managed care operation for pharmacy services.

\section{PHARMACY BENEFIT AND FORMULARY MANAGEMENT}

Historically, MHCPS negotiated capitated contracts with business partners as the preferred method of reimbursement; however, this method is not universal. MHCPS has evaluated and used other forms of payment, from traditional feefor-service to carve-out contracts for specific types of medications or patient conditions.

The MHCPS receives a capitated payment (per member per day) for all pharmacy services provided to inmates and youth offenders covered by MHC. The covered population includes $<28,000$ inmates in 31 TDCJ and TYC facilities throughout West Texas, including 1,100 inpatient psychiatric beds and ambulatory psychiatric programs. MHCPS faculty in a carve-out model staff a 50-bed medical/surgical facility, including a 33-bed dialysis center and specialty medical clinics. No copayment requirements or limitations on the number of prescriptions per "member" exist. Because of the wide range of acute and chronic conditions observed in these populations, drug utilization review is essential for quality of care and for detecting shifts in prescribing that could have an adverse financial impact. All faculty employed through the MHCPS have responsibilities for utilization review and nonformulary drug request evaluation.

Financial and utilization reports are analyzed monthly to determine permember per-day costs by facility. Trend analysis provides information to the MHCPS management team to develop target drug utilization programs. Where costs for specific procedures or conditions can be identified (oncology, dialysis, etc.) MHCPS may consult with other School of Pharmacy faculty to optimize drug therapy.

Nonformulary drug usage in correctional facilities is managed by intervening with the prescriber. Once notified of a nonformulary request, faculty review the patient care profile, consult with care providers at the unit, and notify the prescriber electronically of the approval of the nonformulary product or the suggested alternative medications. Once an order is clarified, designated individuals at the prison unit will transmit the medication order to a central drug distribution center subcontracted by the MHCPS. Monthly data on nonformulary requests and their disposition are analyzed for trends and are reported to the regional pharmacy and therapeutics committee (R-P\&T).

All medication orders are screened electronically at the drug distribution center for interactions or other clinical problems. If a problem with a medication order is detected, information about the order is entered into a database and relayed to MHCPS. MHCPS faculty review a report from the database and consult with the care providers to resolve the issue.

Faculty are integrated into the various administrative committees charged with responsibility for medication use policy development and/or approval. 
The R-P\&T committee meets quarterly to address issues of importance to the correctional and youth offender programs in West Texas. Recommendations from this committee are forwarded to the TDCJ pharmacy and therapeutics committee (TDCJ-P\&T). The TDCJ-P\&T committee consists of representatives from TDCJ Health Services, AHSC, and pharmacy programs that provide care at the correctional facilities. ${ }^{3}$ The committee addresses system-wide issues including maintenance of the formulary, implementation of medication usage policies, approval of therapeutic guidelines, and approval of system-wide medication usage evaluation studies.

\section{INTEGRATING EDUCATION AND PRACTICE}

The School of Pharmacy receives significant programmatic benefit from the contracts negotiated by the MHCPS. The chronic diseases and comorbidities within correctional health practice provide faculty with clinical care challenges as well as excellent educational case materials. Faculty have excellent practice relationships with other care providers in the facilities where MHCPS is responsible for pharmaceutical care. Since 1997, the Texas Board of Medical Examiners and the Texas Board of Pharmacy have permitted drug therapy management under protocol. The School of Pharmacy has encouraged its faculty to develop collaborative agreements with physicians, and they have been very successful in establishing these types of practices.

MHCPS faculty must incorporate clerkship and other experiential training into their daily work activities in contracted sites. The School of Pharmacy curriculum includes several courses to develop pharmaceutical care skills. Under an educational affiliation agreement with TDCJ and MHC, students under the direct supervision of the faculty gain experience in basic clinical skills such as patient assessment and documentation. Selective ambulatory care rotations are also available to fourth-year clerkship students.

MHCPS administrative and clinical faculty are also involved in the didactic portion of the curriculum, although the time for these activities is limited because of the requirements of the service contracts. Clinical faculty primarily teach basic clinical skills and introductory courses in pharmaceutical care. Administrative faculty teach practice management. The active participation of the MHCPS staff in didactic teaching brings "real-life" credibility to the course.

\section{SUMMARY}

Practice partnerships between academic pharmacy programs and public or private business entities can provide significant benefits for both. While acknowledging the potential financial benefit, the greater benefit may be teaching, practice, and research opportunities for the faculty; however, to minimize the risk associated with any business venture, schools of pharmacy must assess the types of services to be provided by the faculty and how those services fit into the educational mission before creating an entrepreneurial vision. A worstcase scenario can quickly develop if the faculty does not perceive the service requirements of contracts, especially capitated managed care contracts, as an extension of their educational duties.

Roland A. Patry, Dr. P.H., FA.S.H.P. Professor of Pharmacy Practice and Associate Dean for Patient Care Services

Butch Habeger, M.B.A., F.A.S.H.P. Assistant Professor of Pharmacy Practice and Chief, Managed Health Care Pharmacy Services Texas Tech University Health Sciences Center School of Pharmacy

\section{A References}

1. Seals TD, Keith MR. Influence of patient information leaflets on anticonvulsant drug compliance in prison. Am J Health-Syst Pharm 1997; 54: 258587.

2. Keith MR, Coffey EL. Clinical training program for distributive pharmacists. Am J Health-Syst Pharm 1997; 54: 674-77.

3. Keith MR. A diabetes disease management plan in a correctional staff model HMO. P\&T 1999; 24: 227-32, 235-37. 